\title{
License Vehicle Plates Localization Using Maximum Correlation
}

\author{
Regis C.P. Marques, Fátima N.S. Medeiros, \\ Jilseph Lopes Silva, and Cassius M. Laprano \\ UFC- DETI \\ Campus do PICI, Bloco 705 \\ 60455-760 - Fortaleza, CE, Brazil \\ \{regismarques, fsombra, jilseph, cassiusml\}@deti.ufc.br \\ http://www.gpi.deti.ufc.br
}

\begin{abstract}
In this paper we propose an automatic method to locate Brazilian license plates in digital images acquired from a real monitoring traffic system. The approach is based on the correlation between lines of the preprocessed image and a square wave form that resembles lines in the region of the pattern plate. The maximum correlation points out the horizontal and vertical crossing axes over the plate. Prior to locating plates, the post processing Min/Max scheme is applied to the binary images in order to diffuse segmentation remnant noise.
\end{abstract}

\section{Introduction}

Many intelligent systems have been developed for traffic control and monitoring system in the last decade. Applications such as detection of irregular vehicles, parking and toll control, license plates location and reading are spread all over the world. These applications are based on automatic recognition of the license plates.

Comelli et al. [1] presented a system to recognize Italian license plates passing through a tollgate. The recognition system consisted of three main phases as follows: plate location, image preprocessing and characters recognition. Fig. 1 exhibits the modules that compounds this system. The preprocessing module consisted of conventional filtering and enhancement techniques and the characters within the plates were recognized by using a combination of operators based on pattern matching and templates. The algorithms were tested on more than three thousand real images with a recognition rate close to $91 \%$. In [2] Naito and Tsukada proposed a robust plates recognition system. This system is capable of capturing fine image under bad illumination conditions, from twilight up to noon in the sunshine, capturing non-blurred moving vehicle images and recognizing plates even being inclined. In this system, even if the position of TV camera varied widely over $97 \%$ of the license plates could be recognized successfully [2]. Naito and Tsukada [2] evaluated the license plates location in binary images instead of using gray level images in order to simplify this task. Inspired in this idea we propose in this paper an algorithm to locate license plates in binary images. Our proposed method concerns the modules inside the dotted lines in Fig.1. It was developed to locate Brazilian license plates in digital images, although it is potentially applicable to other foreign plates. 


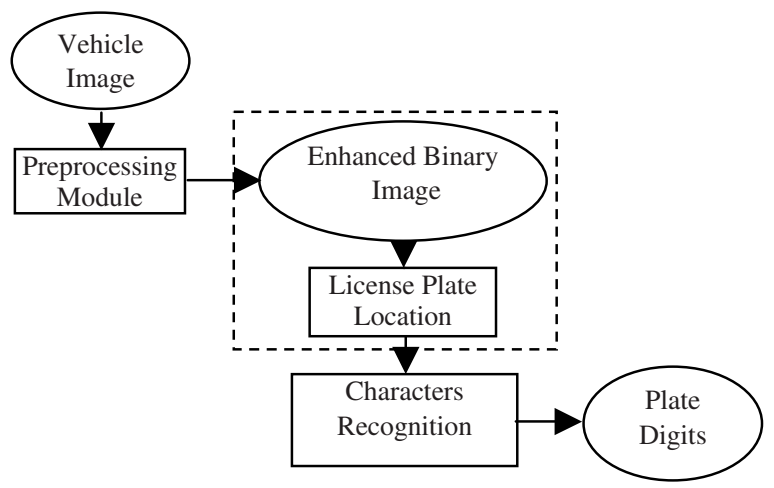

Fig. 1. Vehicle plate recognition system.

An image-based recognition system proposed in [6] applies neural network for automatic vehicle plate recognition. It consists mainly of two processes: the training process and the recognition process. In the former the database of coded plates characters is built and the neural networks are trained to recognize. The latter includes the vehicle plate localization, plate binarization, symbol segmentation, coding of segmented symbols and character recognition. The system was assessed according two aspects such as: the ability to estimate plate position and the plate characters recognition rate.

This paper is organized as follows. Section 2 describes the proposed methodology used to locate license plates. In Section 3 the experimental results are presented and Section 4 summarizes the conclusions.

\section{The Proposed Methodology}

This paper proposes an algorithm to locate license plates in moving or parked vehicles images. The method achieves the maximum correlation operator between the lines of the binary image and a wave form that resembles the pattern plate. In order to improve the segmentation process we apply the Min/Max method proposed by Malladi and Sethian [3] to remove the remnant noise of the segmentation process.

The background concepts on which the proposed method is based on such as segmentation, the Min/Max post-processing scheme and the correlation approach are described in the following.

\subsection{Image Segmentation}

Segmentation is an important task in systems based on digital image processing. Traditionally, thresholding segmentation methods [5] are very popular and computationally efficient in case of bimodal histograms. When these methods are extended to multimodal histograms images they tend to be computationally expensive and inaccurate. However, it is not the aim of this paper to develop or improve segmentation methods. Therefore a simple bilevel thresholding method is used to generate a binary image of the license plate pointing out it from the background. 


\subsection{The Min/Max Post-processing Method}

Level set methods are interactive schemes for noise removal and image enhancement. Some algorithms based on these schemes require a stopping criterion due to Grayson's theorem that says the contour shrinks to zero and disappears [4].

In [3] was proposed a function called Min/Max that switches between removing noise and maintaining essential image properties. It means that the correct curvature flow is based on neighbourhood characteristics. The Min/Max function is presented in Equation (1), where Ave is defined as the average value of $I$ in a disk of radius $R$ centered around the point $(x, y)[4]$.

$$
F_{\min / \max }=\left\{\begin{array}{lll}
\min (k, 0) & \text { if } & A v e_{I(x, y)}^{R}<0 \\
\max (k, 0) & \text { if } & A v e_{I(x, y)}^{R} \geq 0
\end{array} .\right.
$$

The radius $R$ implies that the $R$ th pixels order structures are diffused into the background values. In the test images, the noise is characterized by small structures of first order. Due to this, the radius $R=1$ was chosen in order to diffuse remnant small structures from the segmentation process.

Fig. 2(a) displays a binary image of a vehicle plate and Fig. 2(b) shows its enhanced version applying the Min/Max scheme. As a consequence of the postprocessing task it can be observed a visual improvement in the segmented image. It is worth noting that the segmentation remnant noise in Fig. 2(a) can affect the localization algorithm performance. Thus, the good quality of the images is assured by the post-processing task leading to accurate results.

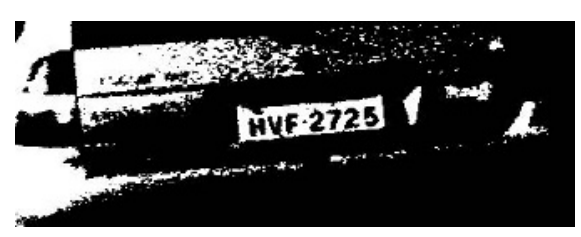

(a)

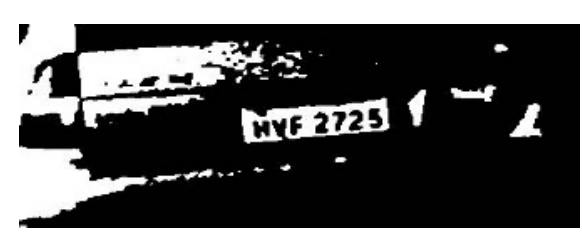

(b)

Fig. 2. (a) Segmented plate image and (b) the enhanced version.

\subsection{The Proposed Method Based on Maximum Correlation}

To locate plates the proposed method calculates the correlation between the locally enhanced binary image (img) and a 1-D square wave function, with period $p$ and length $l(f(p, l))$. The use of a square wave form is justified by the amplitude patterns found in lines over the plate characters region in the binary image. These patterns are shown in Fig. 3, where the central region exhibits the plate patterns.

\section{The Location Process Using the Square Wave Function}

The period of the square wave function is defined as being the half of width of the plate character and the length of the convolution mask $l$ is determined by the width of the plate (Fig. 4). 


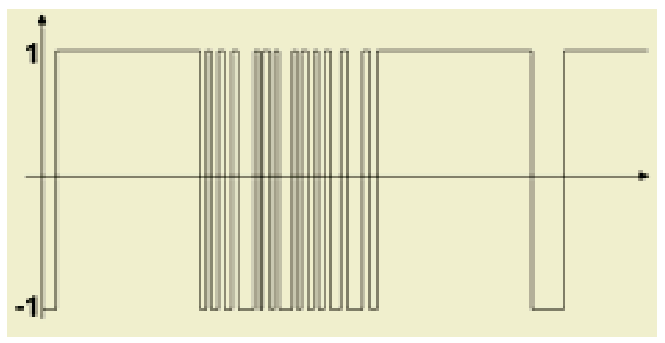

Fig. 3. The pattern line of a plate image.

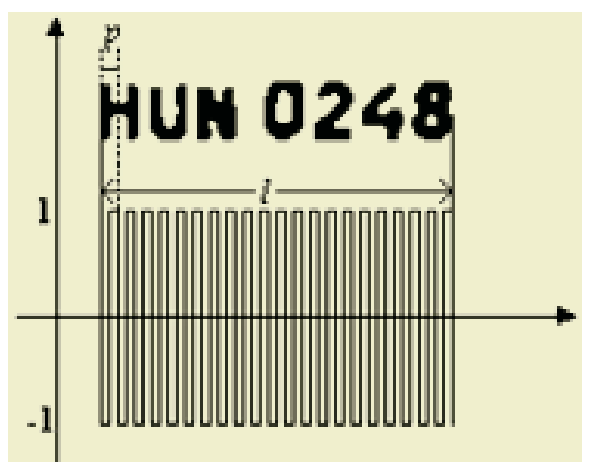

Fig. 4. The square wave function.

Once defined the wave form pattern, the correlation matrix is calculated and the maximum value in it points towards the plate location. The coordinates of the maximum correlation are used to indicate the center of the crossing axes in the located plate. The mathematical expression that defines it is given by:

$$
\text { PlateLocation }=\max \{\operatorname{img} * f(p, l)\} .
$$

where max indicates the maximum correlation value in the correlation matrix, and $\{*\}$ indicates the convolution operator.

Fig. 5 presents the correlation result between a 1-D square wave function and the wave form in Fig. 6.

\section{Experimental Results}

The algorithm was applied to a set of 40 real test images. The database was divided into group 1 and group 2. The former consisting of 7 parked vehicles images and the latter consisting of 33 moving cars images taken by a real monitoring traffic system. Fig. 6 shows a parked vehicle image and Fig. 7 shows the located plate. Fig. 8 and Fig. 9 illustrate the original vehicle image and the incorrect plate location when applied to a moving car image. Fig. 11(a) and Fig. 13(a) illustrate the differences be- 
tween the images that were post-processed and the ones that were not. The use of the post-processing Min/Max scheme improved the performance of the localization algorithm as display Fig. 11(a) and Fig. 13(a). The correct plate location corresponds to the horizontal and vertical axes crossing and it was obtained an overall error rate below $3 \%$ using the set of test images.

Different parameters $p$ and $l$ were set to parked and moving vehicles images, since these values depend on the distance that the pictures are taken by the acquisition system. For parked vehicles images $p=10$ and $l=200$ (pixels), for moving cars images $p=15$ and $l=200$ (pixels).

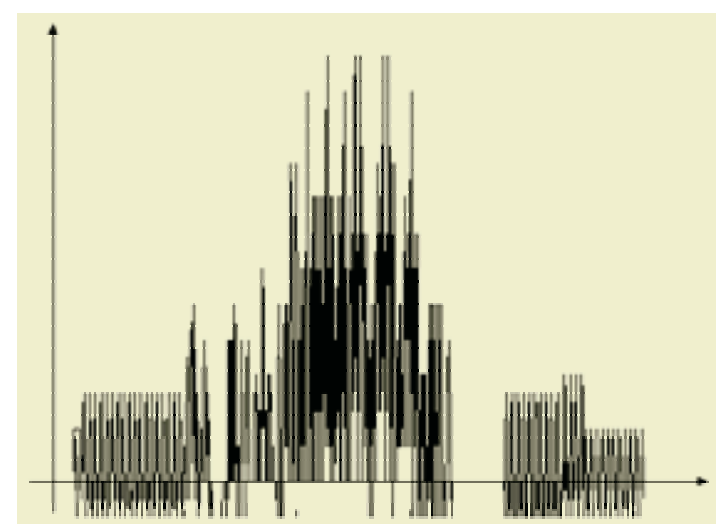

Fig. 5. The correlation result.

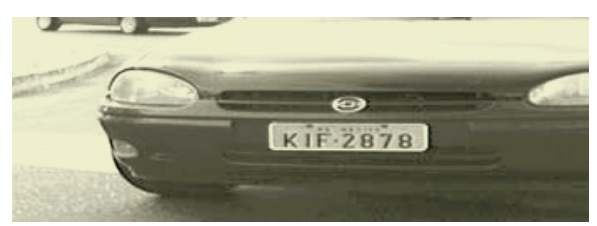

Fig. 6. Parked vehicle image.

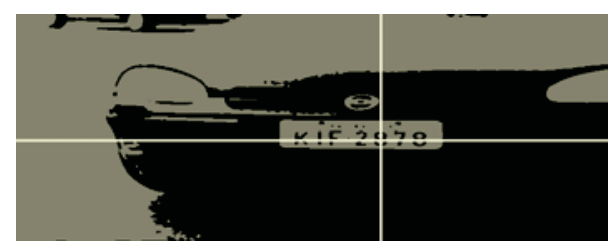

Fig. 7. The located plate.

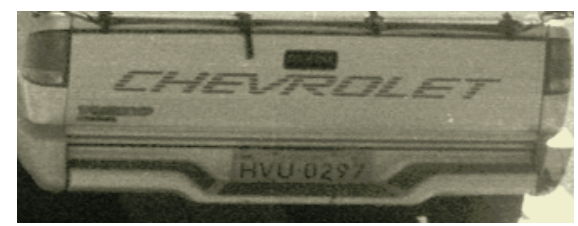

Fig. 8. Moving vehicle image. 


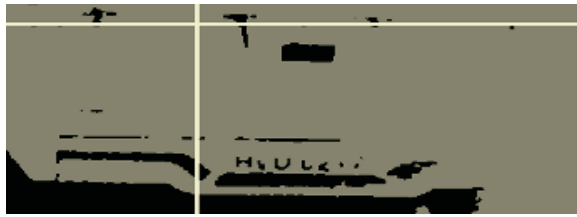

Fig. 9. Incorrect located plate.

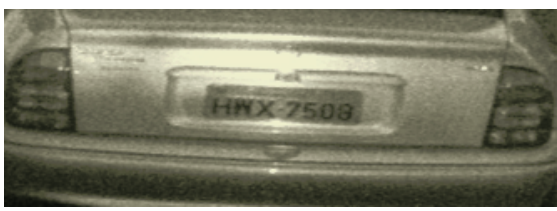

Fig. 10. Moving vehicle image. (Acquisition system in an indirect look).

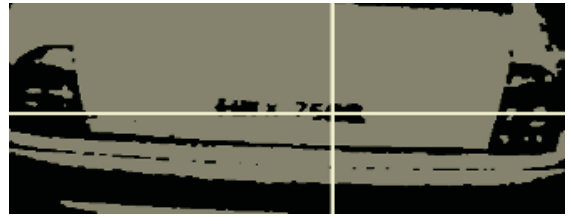

(a)

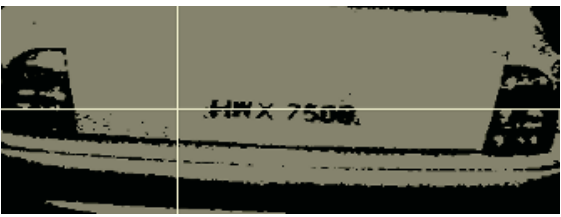

(b)

Fig. 11. (a) Located plate using the Min/Max scheme and (b) not using it.

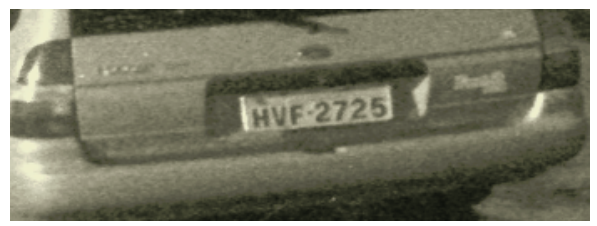

Fig. 12. Moving vehicle image. (Acquisition system in a direct look).

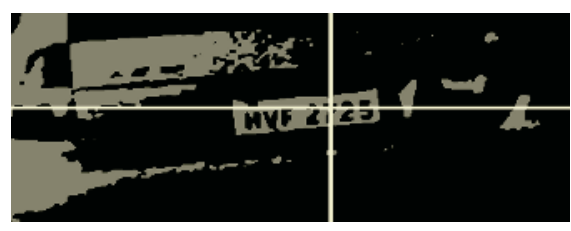

(a)

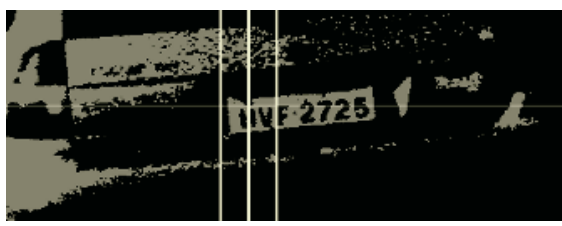

(b)

Fig. 13. (a) Located plate using the Min/Max scheme and (b) not using it.

\section{Concluding Remarks}

The test images used in this paper were segmented simply employing a threshold segmentation method. The threshold value was not automatically adjusted for each 
image because it is difficult to set it for any kind of gray level image. To improve the proposed vehicle plates localization method, the binary images were enhanced by applying the suitable Min/Max scheme. This post-processing algorithm eliminated the remnant noise in the segmented vehicles images and provided reasonable localization results.

The preliminary tests of the algorithms were taken on the set of parked vehicles images producing good results as presents Fig. 7. Furthermore, the most relevant results of the algorithms concerns the moving car images taken by a real monitoring traffic system in different illumination conditions and car positions. Neither the parameters of the square wave function nor the image position were changed during the tests.

The critical point of the proposed method is the segmentation method. As displays Fig. 14 the plate localization result was influenced by the bilevel thresholding scheme used to segment the image. Thus, further developments will focuse segmentation improvements to overcome these kind of difficulties. It is worth noting that the algorithm was adjusted to the Brazilian plates, thus the wave form depends on the national plate pattern and the acquisition system of the license vehicle plates. Therefore, it can be adjusted to different plate patterns.

\section{References}

1. Comelli, P., Ferragina, P., Stabile, M. N., Stabile, F.: Optical Recognition of Motor Vehicle License Plates. IEEE Transactions on Vehicular Technology. Vol. 44(4), (1995) 790-799

2. Naito, T., Tsukada, T., Yamada, K., Kozuka, K., Yamamoto, S.: Robust License-Plate Recognition Method for Passing Vehicles Under Outside Environment. IEEE Transactions on Vehicular Technology. Vol. 49 (6) (2000) 2309-2319

3. Malladi, R., Sethian, J. A.: Image Procesing: Flows under Min/Max Curvature and Mean Curvature. Graphical Models and Image Processing. Vol. 59 (2) (1996) 127-141

4. Sethian, J. A.: Level Set Methods and Fast Marching Methods: Evolving Interfaces in Computational Geometry-Fluid Mechanics. 2nd edn. Computer Vision, and Materials Science. Cambridge University Press. New York (1996)

5. Pratt, W. K.: Digital Image Processing. 2nd edn. John Wiley \& Sons. New York (1991)

6. Vázquez, M. Nakano, M., Pérez-Meana, H.: Automatic System for Localization and Recognition of Vehicle Plate Numbers. Journal of Applied Research and Technology. Vol. 1 (1) (2003) 63-77 